\title{
Application of CLARITY to Investigate the 3D Architecture of 14-3-3 Zeta Protein in AD
}

\author{
Honghong Song ${ }^{1}$, Juan Feng ${ }^{1,}$, Jian $\mathrm{Li}^{2}$, Yang Xia ${ }^{1}$, Dezhong Yao ${ }^{1}$ \\ ${ }^{1}$ College of Life Science and Technology, University of Electronic Science and Technology of China, Chengdu, China \\ ${ }^{2}$ College of Life Science, Beijing University of Chinese Medicine, Beijing, China
}

\author{
Email address: \\ fengjuan@uestc.edu.cn (Juan Feng) \\ ${ }^{*}$ Corresponding author
}

\section{To cite this article:}

Honghong Song, Juan Feng, Jian Li, Yang Xia, Dezhong Yao. Application of CLARITY to Investigate the 3D Architecture of 14-3-3 Zeta Protein in AD. International Journal of Biomedical Science and Engineering. Vol. 5, No. 6, 2017, pp. 68-71. doi: $10.11648 /$ j.ijbse.20170506.12

Received: July 30, 2017; Accepted: September 25, 2017; Published: December 6, 2017

\begin{abstract}
CLARITY (Clear Lipid-exchanged Acrylamide-hybridized Rigid Imaging/ Immunostaining/ in situhybridization-compatible Tissue hydrogel) is a powerful, innovative, whole brain-clearing technology, and it has been successfully combined with the immunofluorescence staining to achieve the $3 \mathrm{D}$ visualization of some proteins or cells in $\mathrm{mm}$-thick brain tissue or even the intact brains. These 3D information help to gain deeper understanding on the pathologic mechanism of some neuronal diseases (for example Parkinson and Alzheimer). 14-3-3 zeta is a highly-expressed protein in Alzheimer's disease (AD) brain, which was closely related with the formation of Tau aggregation and neurofibrillary tangles. However, little useful information has been available concerning the 3D architecture of 14-3-3 zeta in AD disease. In this paper, the transgenic $\mathrm{AD}$ mice were used and the $1 \mathrm{~mm}$-thick brain slices were passively clarified. Immunofluorescence staining results showed that different from the control group, 14-3-3 zeta was mainly present around the hippocampus in AD mice. Additionally, the morphology of 14-3-3 zeta protein was filamentous with different lengths. This result will be helpful for exploring the in vivo role of 14-3-3 zeta protein during the progression of AD.
\end{abstract}

Keywords: AD, CLARITY, 14-3-Zeta Protein, Immunofluorescence

\section{Introduction}

CLARITY is one new brain-clearing technology discovered in 2013. Different from other clearing methods, including BABB [2], 3DISCO [3], Sca/e [4], See DB [5] and Cubic [6], SDS detergent is utilized in CLARITY to remove the lipids rich in brain tissue. Because SDS can also wrap and cause the protein loss, the researchers introduce the hydrogel monomer that can react with proteins into the brain tissue. As these monomers are polymerized, the proteins are fixed to the the hydrogel matrix [7, 8]. Following these treatments, the brain tissues become transparent because the light scattering is dramatically reduced with the presence of fewer lipids. Meanwhile, the antibodies have more accesses to diffuse into the brain tissues containing the porous hydrogel matrix. These advantages have attracted more and more attention from all over the world. Some researchers aimed to optimize the
CLARITY condition $[9,10]$ or to extend CLARITY to other tissues or fields $[11,12,13,14$, and 15]. Other researchers have successfully applied CLARITY to visualize the $3 \mathrm{D}$ architecture of some molecules or cells that participate in some neuronal diseases, including AD and Parkinson [16, 17]. In 2014 Ando $\mathrm{K}$. et al explored the 3D distribution of $\mathrm{Ab}$, phosphorylated Tau and neurofibrillary tangles (NFT) in the $500 \mu \mathrm{m}$ frontal cortex of AD people by combing CLARITY and immunohistochemistry methods [16]. However, no such information has been reported for other proteins involved in AD.

It is known that 14-3-3 zeta protein is one member of 14-3-3 protein family. In the recent years, people pay much attention to the role of 14-3-3 zeta protein in cancer therapy and the neurogenesis [18, 19, 20, and 21]. In AD disease 14-3-3 zeta was found to regulate the phosphorylation of Tau and affect the aggregation of Tau [22]. Nevertheless, these studies were almost done in vitro. Little useful information has been 
available concerning the in-vivo studies of 14-3-3 zeta, for example the $3 \mathrm{D}$ distribution, as well as its regulation on phosphorylated Tau in vivo. In this paper, the transgenic AD mice were used as model. CLARITY was done to make the mm-thick brain slices become transparent. Subsequently, the clarified brain section was incubated with anti-14-3-3 zeta primary antibody and secondary antibody labeled by Atto- 488 fluorescence dye. The fluorescent imaging result showed that 14-3-3 zeta was distributed around the hippocampus region, and 14-3-3 zeta exhibited filamentous structure.

\section{Main Body}

\subsection{Animals}

In this work, the transgenic APP-overexpressing mice were used as AD models.

\subsection{CLARITY}

According to the literature [7], CLARITY was performed on $\mathrm{AD}$ mice brain using passive diffusion method in the present work. The details are shown as follows. In the first step, the AD and control group mice were deeply anesthetized using $10 \mathrm{~mL}$ of $10 \%$ hydrated chloralhydrate. Subsequently, after the mice were transcardially perfused using $100 \mathrm{~mL}$ of ice cold $0.9 \% \mathrm{NaCl}$ solution, $40 \mathrm{~mL}$ of the acrylamide hydrogel solution was perfused containing $4 \%$ paraformaldehyde (PFA), $4 \%$ acrylamide, $0.125 \%$ bisacrylamide and $0.25 \%$ the initiator VA-044. In the next step, the intact brain was extracted immediately and immersed in the hydrogel solution at $4^{\circ} \mathrm{C}$ for 3 days in the darkness. After oxygen was removed by bubbling argon gas, the hydrogel was polymerized by heating the solution at $37^{\circ} \mathrm{C}$ for 3 hours. The $\sim 1 \mathrm{~mm}$-thick brain slabs were obtained using mouse abrasives, and then were clarified using the clearing solution $(200 \mathrm{mM}$ boric acid, $4 \% \mathrm{w} / \mathrm{v}$ SDS, $\mathrm{pH} 8.5)$ at $37^{\circ} \mathrm{C}$ for 1 week. Finally, the clarified slices were submerged in $1 \times$ PBS buffer containing $0.1 \%$ Triton $\mathrm{X}-100$ (denoted as $\mathrm{PBST}$ ) for at least $2 \mathrm{~d}$ so as to remove the clearing solution.
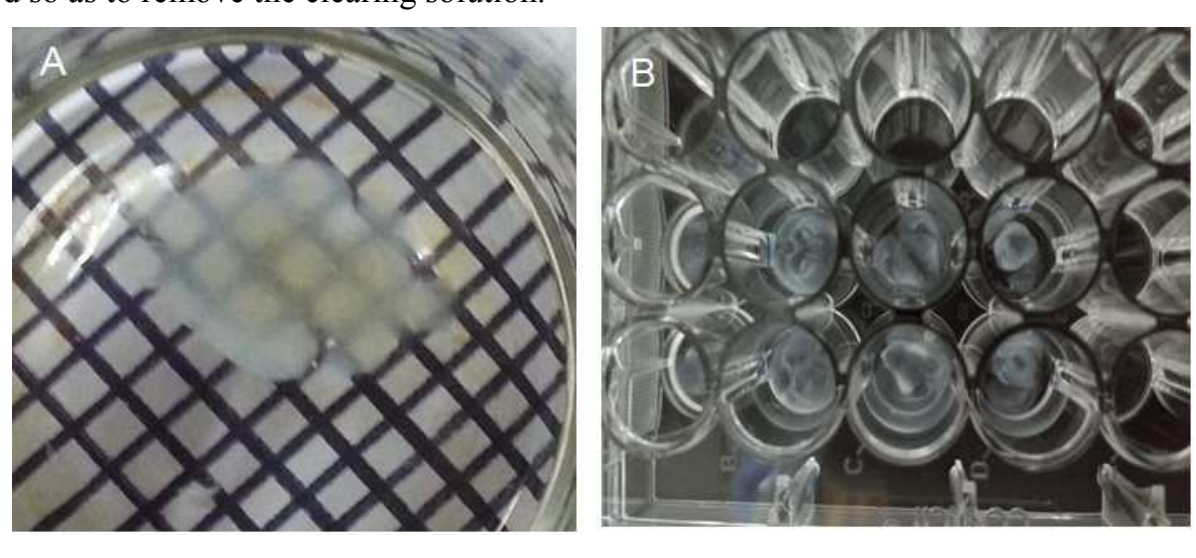

Figure 1. Photographs of the clarified intact brain (A) and 1mm-thick brain slice (B) of AD mice, and they were obtained by electrophoresis and passive diffusion method, respectively.

In the next work the immunofluorescent staining was conducted on the clarified slices using Goat-anti human 14-3-3

\subsection{Immuostaining and Imaging}

After the slices were transferred into 48-well plates, the blocks were stained by Goat-anti Human 14-3-3 zeta primary antibody (Pierce, MA1-045) at $37^{\circ} \mathrm{C}$ for 3 days. After 3-day incubation, the slices were washed in PBST for 2 days. After this step, Alexa Fluor ${ }^{\circledR} 488$ Donkey-anti Goat (Life Technologies, A-11008) secondary antibody was used to stain the blocks at $37^{\circ} \mathrm{C}$ for 3 days and the excess antibody was washed out in PBST for 2 days. During this step, the primary and secondary antibodies are diluted into 1:100 with PBS solution. Dapi was also added to stain the nucleus. Following this step, the slabs were immersed in $85 \%$ glycerol mounting solution to achieve the refractive index matching. The 3D fluorescence imaging was done using Leica excitation wavelength was fixed at $800 \mathrm{~nm}$ for Alexa 488-labeled antibody and $700 \mathrm{~nm}$ for Dapi, respectively. The Z-stack images were re-constructed using Imaris v7.4.2. software (Bitplane).

\section{Details}

In this paper, the tail suspension test and morris water maze were done to ensure that the 0.5 -year old transgenic mice had suffered from AD (data not shown). Next, one electrophoresis setup was self-built to clarify the intact mice brain. As shown in Figure 1A, the AD mice brain had become transparent, but there appeared some yellow spots in the middle part of the brain tissue. The reason might be due to the presence of haemosiderin or lipofuscin-type pigments, as reported in the literature [17]. Here we can not exclude another possibility, i.e. the heat produced during SDS-electrophoresis might result in the denaturation of some proteins. As a result, the passive diffusion method was finally used instead of SDS-electrophoresis setup in this work. It was found that it took at least one month to clarify the intact brain, while the mm-thick brain slabs could become transparent within one week. The clarified slabs were shown in Figure 1B. LAS 4.0 two-photon confocal fluorescence microscopy. The 
was used to stain the cellular nucleus. Figure 2A showed the fluorescence imaging results of the clarified mm-thick $\mathrm{AD}$ brain block following the excitation at $800 \mathrm{~nm}$. We found that 14-3-3 zeta protein was far away from the hippocampus region shown by the arrow. No similar result was observed for the control group (seen from Figure 2C), where 14-3-3 zeta was detected in the cerebral cortex region. This spatial distribution was also different from the previous studies, in which 14-3-3 zeta was located to the neurons in the human cerebral cortex
[23]. It might be due to the time-dependent pattern change of 14-3-3 zeta protein during the progression of AD. Additionally, the $3 \mathrm{D}$ architecture of $14-3-3$ zeta protein was analyzed by $3 \mathrm{D}$ reconstruction within $200-\mu \mathrm{m}$-thick range. As demonstrated in Figure 2B, 14-3-3 zeta protein in $\mathrm{AD}$ mice appeared to be filamentous and heterogeneous: some were short and discontinuous; some were long and continuous. For the time being, it is unclear what is the role of 14-3-3 zeta with different length in $\mathrm{AD}$ mice brain.
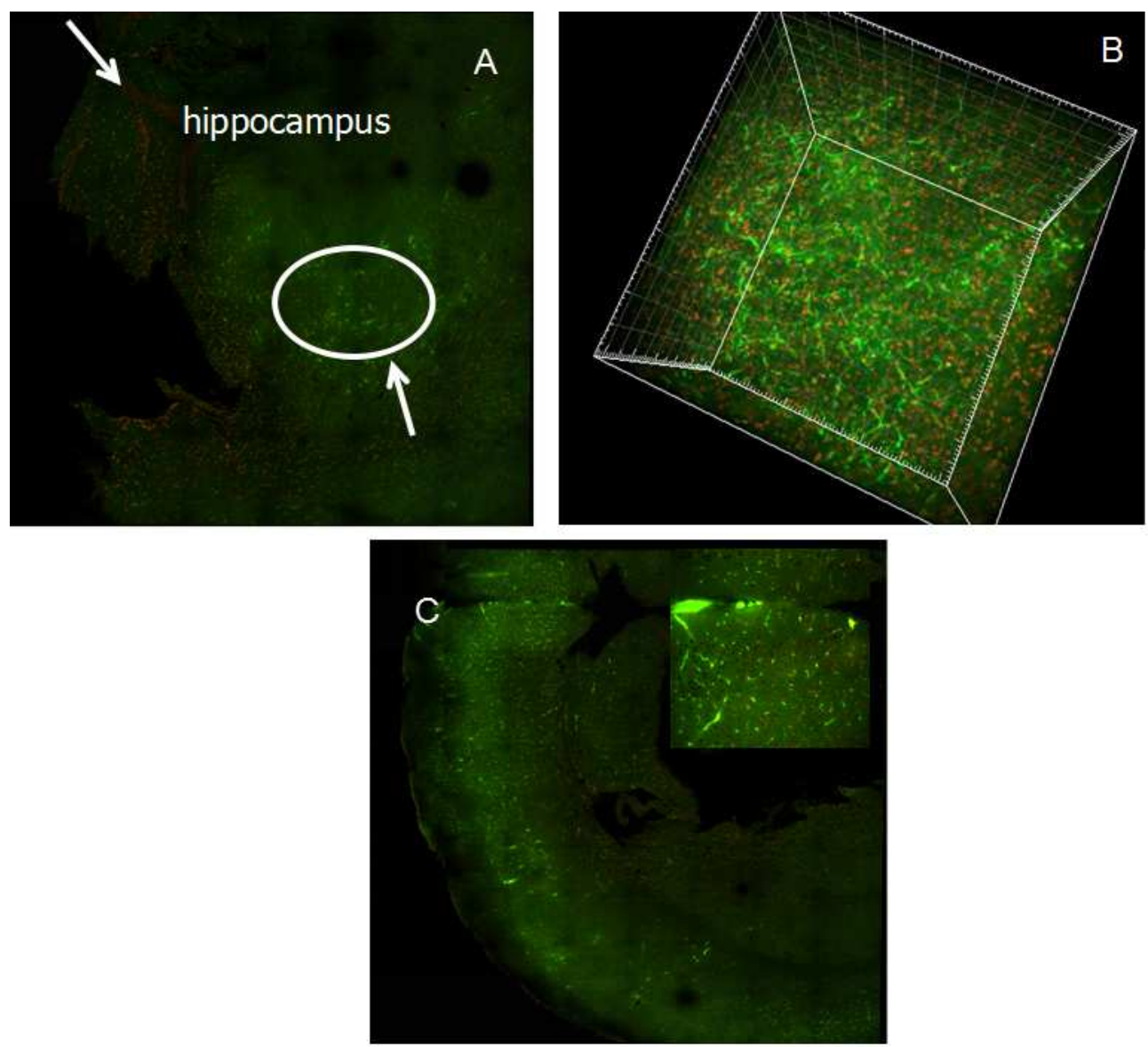

Figure 2. Immunostaining results of 1 mm-thick AD mice brain slice (A), the $3 D$ reconstruction of $200 \mu \mathrm{m}$ slice (B) and immunostaining results of control group mice $(C)$.

\section{Conclusion}

In this work we clarified the mm-thick brain slices of the transgenic $\mathrm{AD}$ mice by using passive diffusion method. Immunofluorescence labeling was performed on these mm-thick sections in PBS buffer at $37^{\circ} \mathrm{C}$. The observed strong fluorescence signals indicated that the 1:200 diluted primary and secondary antibody had smoothly diffused into the porous brain tissue. Furthermore these antibodies targeted 14-3-3 zeta protein with high specificity. Immunofluorescence staining results showed that (1) Unlike other results, 14-3-3 zeta protein was mainly distributed around the hippocampus in AD mice brain. It probably indicated that the position alteration of 14-3-3 zeta protein might be implicated in the progression of AD. More work needs to be done for exploring whether the localization of 14-3-3 zeta protein is time-dependent during the development of $\mathrm{AD}$. (2) The 3D reconstruction results of fluorescence imaging showed that the morphology of 14-3-3 zeta protein was filamentous with different lengths. In the further work, double or triple immunofluorescence staining would help to investigate if the interaction between 14-3-3 zeta with other proteins (for example Tau) should be responsible for the length variation of 14-3-3 zeta protein.

\section{References}

[1] Chung K, Wallace J, Kim SY, et al. Structural and molecular interrogation of intact biological systems. Nature, 2013, 497(7449): 332-337.

[2] Dodt HU, Leischner U, Schierloh A, et al. Ultramicroscopy: three-dimensional visualization of neuronal networks in the whole mouse brain. Nat Methods., 2007, 4(4): 331-336. 
[3] Ertürk A, Becker K, Jährling N, et al. Three-dimensional imaging of solvent-cleared organs using 3DISCO. Nat Protoc., 2012, 7(11):1983-1995.

[4] Hama H, Kurokawa H, Kawano H, et al. Sca/e: a chemical approach for fluorescence imaging and reconstruction of transparent mouse Brain. Nat Neurosci., 2011, 14(11): $1481-1488$.

[5] Ke MT, Fujimoto S, Imai T. See DB: a simple and morphology-preserving optical clearing agent for neuronal circuit reconstruction. Nat Neurosci. 2013., 16(8): 1154-1161.

[6] Susaki EA, Tainaka K, Perrin D, Whole-brain imaging with single-cell resolution using chemical cocktails and computational analysis. Cell, 2014, 157(3): 726-739.

[7] Chung K, Deisseroth K. CLARITY for mapping the nervous system. Nat Methods., 2013, 10: 508-513.

[8] Chung K, Wallace J, Kim SY, et al. Structural and molecular interrogation of intact biological systems. Nature, 2013, 497: 332-337.

[9] Bastrup J, Larson PH. Optimized CLARITY technique detects reduced parvalbumin density in a genetic model of schizophrenia. Journal of neuroscience methods, 2017, 283: 23-32.

[10] Deisseroth K. Optical and chemical discoveries recognized for impact on biology and psychiatry. EMBO Reports, 2017, 18(6): 859-860.

[11] Milgroom A, Ralston E. Clearing skeletal muscle with CLARITY for light microscopy imaging. Cell Biology International, 2016, 40(4): 478-483.

[12] Muzumdar MD, Dorans KJ, Chung KM, et al. Clonal dynamics following p53 loss of heterozygosity in Kras-driven cancers. Nature Communications, 2016, 7: 12685.

[13] Ding Y, Lee J, Ma J, et al. Light-sheet fluorescence imaging to localize cardiac lineage and protein distribution. Scientific Reports, 2017, 7: 42209. doi: 10.1038/srep42209.
[14] Leuze C, Aswendt M, Ferenczi E, et al. The separate effects of lipids and proteins on brain MRI contrast revealed through tissue clearing. Neuro Image, 2017, 156: 412-422.

[15] Syed AM, Wilhelm S, Glancy DR. Three-dimensional optical mapping of nanoparticle distribution in intact tissues. ACS Nano, 2016, 10(5): 5468-5478.

[16] Ando K, Laborde Q, Lazar A, et al. Inside Alzheimer brain with CLARITY: senile plaques, neurofibrillary tangles and axons in 3-D. Acta Neuropathol., 2014, 28(3): 457-459.

[17] Liu AK, Hurry ME, Ng OT et al. Bringing CLARITY to the human brain: visualization of Lewy pathology in three dimensions. Neuropathology and applied neurobiology, 2016, 42: 573-587.

[18] Matta A, Siu KW, Ralhan R. 14-3-3 zeta as novel molecular target for cancer therapy. Expert Opin ther Targets., 2012, 16(5): 515-523.

[19] Toyo-oka K, Wachi T, Hunt RF, et al. 14-3-3 $\varepsilon$ and $\zeta$ regulate neurogenesis and differentiation of neuronal progenitor cells in the developing brain. J Neurosci., 2014, 34(36): 12168-12181.

[20] Ding H, Underwood R, Lavalley N, et al. 14-3-3 inhibition promotes dopaminergic neuron loss and 14-3-3 theta overexpression promotes recovery in the MPTP mouse model of Parkinson's disease. Neuroscience, 2015, 307: 73-82.

[21] Omi K, Hachiya NS, Tanaka M, et al. 14-3-3 zeta is indispensable for aggregate formation of polyglutamine-expanded huntingtin protein. Neurosci Lett., 2008, 24(431): 45-50.

[22] Qureshi HY, Li T, MacDonald R et al. Interaction of 14-3-3 zeta with microtubule-associated protein tau within Alzheimer's disease neurofibrillary tangles, Biochemistry, 2013, 52: 6445-6455.

[23] Boston PF, Jackson P, Kynoch PA, Thompson RJ. Purification, properties, and immunohistochemical localisation of human brain 14-3-3 protein. J Neurochem., 1982, 38(5): 1466-1474. 